\title{
Recent results and prospects of rare kaon decay measurements at $\mathrm{LHCb}$
}

\author{
Carla Marin Benito* ${ }^{\dagger}$ \\ University of Barcelona \\ E-mail: cmarindecm.ub. edu
}

Rare $K_{\mathrm{S}}^{0}$ decays are a new area of interest for $\mathrm{LHCb}$. Latest results on the $K_{\mathrm{S}}^{0} \rightarrow \mu \mu$ search are reported using data collected by the LHCb experiment at a center-of-mass energy of $\sqrt{s}=7 \mathrm{TeV}$, corresponding to an integrated luminosity of $1 \mathrm{fb}^{-1}$. The upper limit at $90 \% \mathrm{CL}$ on the branching fraction of this decay is found to be $9 \times 10^{-9}$. This limit is 30 times lower than the previous world best. $\mathrm{LHCb}$ prospects for other $K_{\mathrm{S}}^{0}$ and strange-hadron decays are also reported.

XIIth International Conference on Heavy Quarks and Leptons 2014,

25-29 August 2014

Schloss Waldthausen, Mainz, Germany

* Speaker.

${ }^{\dagger}$ on behalf of the LHCb collaboration. 


\section{Introduction}

Strange mesons have played a major role in the history of particle physics. When first observed, back in the late 1940s, they triggered large interest. Experiments showed that kaons were produced through the strong force but they were not decaying through this interaction and were long-lived particles. This fact helped to understand in a better way the weak interaction, which was pretty unknown at that moment, and motivated the GIM mechanism and the prediction of the c quark [1].

Another key point in the history of kaon physics was the discovery of charge-parity $(C P)$ violation. It was first observed in 1964 in the decay of $K_{\mathrm{L}}^{0} \rightarrow \pi^{+} \pi^{-}$at the Brookhaven National Laboratory [2]. Charge-parity violation was completely unexpected at that moment and its observation triggered many advances in the understanding of particle physics.

Strange-meson decays are very interesting both from the theoretical and experimental point of view. On one side, they are theoretically clean and involve flavour changing neutral currents (FCNC) with the strongest Cabibbo-Kobayashi-Masakawa (CKM) suppression $\left(V_{t s} V_{t d} \sim 10^{-4}\right)$, making them very sensitive to New Physics (NP) effects. From the experimental side, there is a copious production of strange particles at the LHC and the number of possible final states is limited, allowing for clean studies of these decays.

\subsection{LHCb detector for strange decays}

The LHCb detector is a single-arm forward spectrometer described in detail in Ref. [3]. The detector is optimized for the study of $\mathrm{b}$ and $\mathrm{c}$ hadrons. Although strange mesons have in average lower masses and larger decay times, they are produced in copious amounts at the LHC. During 2011, at a center-of-mass energy of $\sqrt{s}=7 \mathrm{TeV}$, the LHC produced $\sim 10^{13} K_{\mathrm{S}}^{0} \mathrm{per} \mathrm{fb}^{-1}$ inside the LHCb acceptance [4].

Due to the larger decay times of strange mesons, many of them decay outside the vertex locator (VELO). This fact implies that the daughter tracks are only detected in the subsequent subdetectors. These tracks are referred to as downstream tracks and have in average worse momentum resolution and thus worse vertex resolution. Tracks that are detected also inside the VELO are referred to as long tracks.

When the decay mother is a charged particle, it leaves also hits in the VELO that can be matched to the downstream tracks. This technique helps to improve a lot the selection of these decays as random combinations of downstream tracks are not pointing to the hits of the mother particle. This technique is shown in more detail in Section 3.3.

\subsection{LHCb trigger for strange decays}

The LHCb trigger [5] consists of a hardware stage, which selects high $p_{T}$ and $E_{T}$ signatures based on the information from the calorimeters and muon chambers, followed by a software stage which after selecting displaced tracks using tracking and vertexing information, performs a full event reconstruction.

In general, the LHCb trigger is not designed to select strange decays, since it takes advantage of the distinct signatures of $b$ and $c$ hadron decays. Nevertheless, in 2011, around $1 / 3$ of the events that passed any trigger decision contained a reconstructible $K_{\mathrm{S}}^{0} \rightarrow \pi^{+} \pi^{-}$. 
During 2011, the di-muon invariant mass requirement in the software trigger was higher than the $K_{\mathrm{S}}^{0}$ mass. Thus, only triggers selecting single muons were useful to select decays like $K_{\mathrm{S}}^{0} \rightarrow$ $\mu^{+} \mu^{-}$. In 2012, a low mass di-muon line compatible with the $K_{\mathrm{S}}^{0}$ mass was included. This resulted in an increase in the trigger efficiency for $K_{\mathrm{S}}^{0} \rightarrow \mu^{+} \mu^{-}$of a factor 3. Additional studies are ongoing to improve this efficiency for LHC Run 2.

2. $K_{\mathrm{S}}^{0} \rightarrow \mu^{+} \mu^{-}$

The $K_{\mathrm{S}}^{0} \rightarrow \mu^{+} \mu^{-}$decay is a FCNC with no tree-level contribution in the Standard Model $(\mathrm{SM})$, with a predicted branching fraction $(\mathrm{BF})$ of $(5.1 \pm 0.2) \times 10^{-12}$ [7]. The decay amplitude of $K^{0} \rightarrow \ell^{+} \ell^{-}$includes a s-wave and a p-wave component. The two amplitudes have different $\mathrm{CP}$, such that CP-conserving contributions to $K_{\mathrm{L}}^{0}$ and $K_{\mathrm{S}}^{0}$ are generated by the s-wave and the p-wave, respectively. Long-distance contributions can lead to both s-wave and p-wave amplitudes but with a negligible CP-violating component. On the contrary, short-distance contributions of the SM type can contribute only to the s-wave amplitude but in this case $\mathrm{CP}$-violating phases are expected to be $O(1)$. For this reason, within the SM, the p-wave term in the $K_{\mathrm{L}}^{0} \rightarrow \mu^{+} \mu^{-}$amplitude can be safely neglected. On the other hand, in the $K_{\mathrm{S}}^{0} \rightarrow \mu^{+} \mu^{-}$case both types of amplitudes need to be considered, making this decay more sensitive to NP contributions.

Within the SM, there are two contributions to the amplitude of this process [6]: the longdistance contribution, which is mediated by virtual photons, and the short-distance, which is mediated by electroweak bosons. The last one is dominated by the $C P$ violating part of the quark level transition $s \rightarrow d ł$, making this decay very sensitive to NP contributions.

From the experimental side, the previous best measurement dates from 1973 when upper limits on $\mathscr{B}\left(K_{\mathrm{S}}^{0} \rightarrow \mu^{+} \mu^{-}\right)<3.1 \times 10^{-7}$ were determined at $90 \%$ confidence level (CL) by an experiment on the CERN PS[8], far from the SM prediction.

\subsection{LHCb analysis}

The LHCb collaboration reported in 2012 an improved measurement of the BR limit for the decay $K_{\mathrm{S}}^{0} \rightarrow \mu^{+} \mu^{-}$[4]. The analyses used $1 \mathrm{fb}^{-1}$ of data collected at a center-of-mass energy of $\sqrt{s}=7 \mathrm{TeV}$.

The $K_{\mathrm{S}}^{0}$ candidates were reconstructed using long track muon pairs coming from the same vertex using the excellent LHCb muon identification, vertex and momentum resolutions. A multivariate algorithm, a Boosted Decision Tree (BDT), was used to reject candidates coming from random combination of two muons and muons resulting from the interaction of particles produced in the $p p$ collision vertex with the material in the region of the VELO.

The $K_{\mathrm{S}}^{0} \rightarrow \pi^{+} \pi^{-}$channel was used as normalization channel. The separation of this decay from the signal channel was studied using $K_{\mathrm{S}}^{0} \rightarrow \pi^{+} \pi^{-}$decays reconstructed and selected in the same way as the signal channel, both in the $\pi^{+} \pi^{-}$and $\mu^{+} \mu^{-}$mass hypotheses. As shown in Fig. 1, the two mass peaks are separated by $\sim 40 \mathrm{MeV} / c^{2}$. This separation, combined with the $\mathrm{LHCb}$ mass resolution of about $4 \mathrm{MeV} / c^{2}$ for such combination of tracks, was used to discriminate between the two channels.

The search region in the di-muon invariant mass was defined between $[492,504] \mathrm{MeV} / c^{2}$. The background level was calibrated by interpolating the observed yield in the mass side-bands to the 


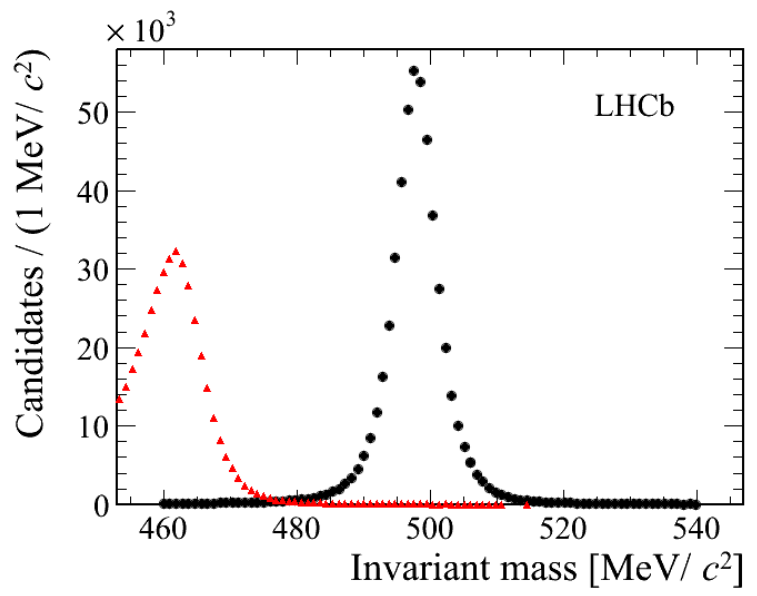

Figure 1: Mass spectrum for selected $K_{\mathrm{S}}^{0} \rightarrow \pi^{+} \pi^{-}$candidates. The points denoted with full circles correspond to the mass reconstructed under the $\pi^{+} \pi^{-}$mass hypothesis for the daughters, while the points denoted with triangles correspond to the mass reconstructed under the $\mu^{+} \mu^{-}$mass hypothesis.

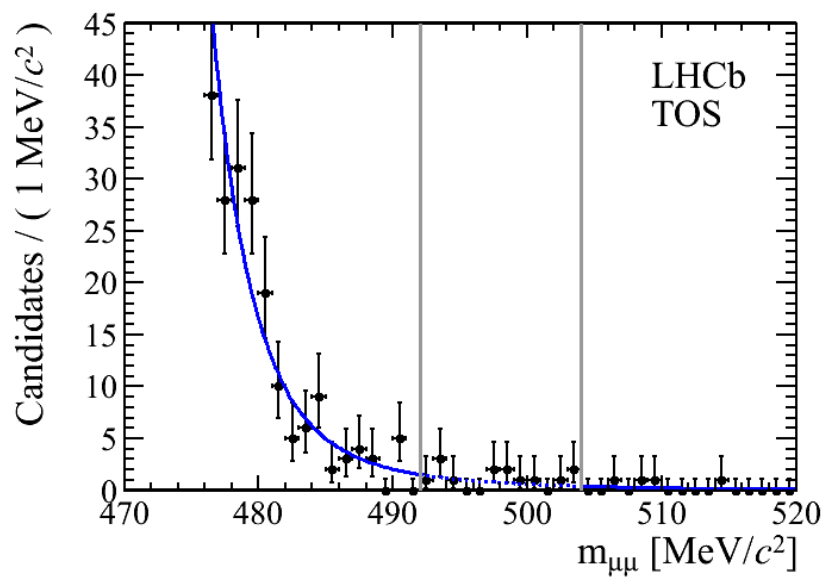

Figure 2: Background model fitted to data events which were triggered on signal (TOS), i.e. the properties of the signal objects were responsible to fire the $\mathrm{LHCb}$ trigger. The vertical lines delimit the search region.

signal region. A model with two components was used: a power law to describe the tail of $K_{\mathrm{S}}^{0} \rightarrow$ $\pi^{+} \pi^{-}$decays where both pions are misidentified as muons and an exponential function describing the combinatorial background. Other sources of background were found to be negligible. The invariant mass distribution of selected candidates is shown in Fig. 2 .

To translate the number of $K_{\mathrm{s}}^{0} \rightarrow \mu^{+} \mu^{-}$signal decays into a branching fraction measurement, the single event sensitivity was normalised to the number of $K_{\mathrm{S}}^{0} \rightarrow \pi^{+} \pi^{-}$events in each of the BDT bins. Using the value of the BF of $K_{\mathrm{S}}^{0} \rightarrow \pi^{+} \pi^{-}$from ref. [9], around $2 \times 10^{-4} \mathrm{SM} K_{\mathrm{S}}^{0} \rightarrow \mu^{+} \mu^{-}$ candidates were expected per BDT bin.

The modified frequentist approach (or CLs method) [10] was used to assess the compatibility of the observation with expectations as a function of the $\mathscr{B}\left(K_{\mathrm{S}}^{0} \rightarrow \mu^{+} \mu^{-}\right)$. The observed distribution of events was compatible with the background-only expectation, as shown in Fig. 3. The measured upper limit is $11(9) \times 10^{-9}$ at $95(90) \%$ confidence level, a factor of thirty below the 


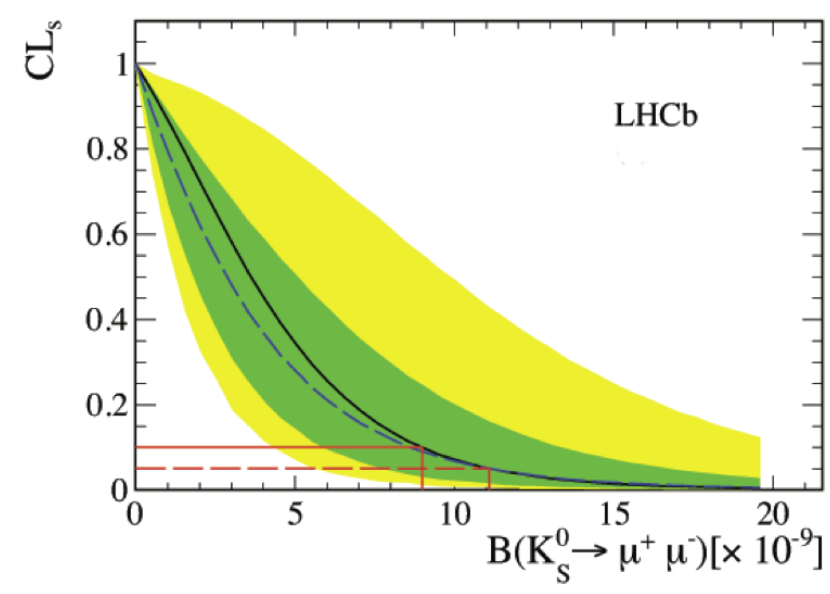

Figure 3: CLs curves as a function of the branching fraction of Ks->mumu. The solid line corresponds to the observed CLs. The dashed line corresponds to the median of the CLs for an ensemble of backgroundalone experiments. The dark band covers 65\% $(1 \sigma)$ of the CLs curves obtained in the background only pseudo-experiments, while the light band covers $95 \%(2 \sigma)$.

previous world best limit.

\section{$2.2 K_{\mathrm{S}}^{0} \rightarrow \mu^{+} \mu^{-}$prospects at $\mathrm{LHCb}$}

LHCb has improved by a factor of 30 the previous world best upper limit measurement but there is still need for improvement to get to the SM prediction of $(5.1 \pm 0.2) \times 10^{-12}$. The most interesting region is below the $10^{-10}$ limit where most new physics models have predictions.

The current limit makes use of only one third of the total amount of data recorded by the LHCb detector in Run 1. In addition, as mentioned in Section 1.2 a threefold improvement in the trigger efficiency was already obtained from the adoption of new trigger strategies in 2012. Some extra gain may be obtained by making use of downstream muon tracks. With these improvements, the LHCb collaboration expects to reach the $10^{-10}$ limit in its upgrade phase, with an expected accumulated luminosity of $40 \mathrm{fb}^{-1}$.

\section{Other rare strange prospects at $\mathrm{LHCb}$}

With the publication of the $K_{\mathrm{S}}^{0} \rightarrow \mu^{+} \mu^{-}$analysis, strange decays have become a new area of interest at $\mathrm{LHCb}$ with several ongoing studies on this field. A selection is reported here.

\section{1 $K_{\mathrm{S}}^{0} \rightarrow \pi^{0} \mu^{+} \mu^{-}$}

The CP-conserving $K_{\mathrm{S}}^{0} \rightarrow \pi^{0} \mu^{+} \mu^{-}$decay is dominated by the long-distance $K^{0} \rightarrow \pi^{0} \gamma^{*}$ contribution. Then, the measurement of this decay can be used to study the structure of the $K^{0} \rightarrow \pi^{0} \gamma^{*}$ form factor. Moreover, the related $K_{\mathrm{L}}^{0} \rightarrow \pi^{0} \mu^{+} \mu^{-}$decay has an indirect CP-violating contribution from the $K^{0} \rightarrow \pi^{0} \gamma^{*}$ component, which can be obtained from the measurement of the $K_{\mathrm{S}}^{0}$ decay. This allows to extract the direct CP-violating contribution of the $K_{\mathrm{L}}^{0}$ one, which can provide input to determine the imaginary part of the element $V_{t d}$ of the mixing matrix. 
The NA48 collaboration reported in 2012 the first observation of this decay [11] and a BR measurement of $\left(2.9_{-1.2}^{+1.5} \pm 0.2\right) \times 10^{-9}$ with an uncertainty of $\sim 50 \%$.

The most challenging issue of this analysis at LHCb is the $\pi^{0}$ reconstruction. Different possibilities have been studied using simulated data and the most feasible option is the reconstruction of $\pi^{0} \rightarrow \gamma \gamma$. Although few $K_{\mathrm{S}}^{0} \rightarrow \pi^{0} \mu^{+} \mu^{-}$events are expected in the $3 \mathrm{fb}^{-1}$ currently accumulated by the $\mathrm{LHCb}$ detector, a measurement may be feasible in the LHCb upgrade, when a huge production of $K_{\mathrm{S}}^{0}$ is expected.

$3.2 K_{\mathrm{S}}^{0} \rightarrow 4 \ell$

The predicted branching fraction of $K_{\mathrm{S}}^{0} \rightarrow 4 \ell$ in the SM are expected to be [12]:

$$
\begin{aligned}
K_{\mathrm{S}}^{0} \rightarrow \text { eeee } & \sim 10^{-10} \\
K_{\mathrm{S}}^{0} \rightarrow \mu \mu e e & \sim 10^{-11} \\
K_{\mathrm{S}}^{0} \rightarrow \mu \mu \mu \mu & \sim 10^{-14}
\end{aligned}
$$

Any deviation from these values may hint to contributions from NP. No experimental results have been reported so far.

For those decays containing electrons in the final state, the reconstruction of the electrons is the most challenging issue at LHCb, due to the energy loss they suffer by Bremsstrahlung. Studies on simulated data allow to extract an expected mass resolution of $\sim 20(\sim 10) \mathrm{MeV} / c^{2}$ for $K_{\mathrm{S}}^{0} \rightarrow$ eeee $\left(K_{\mathrm{S}}^{0} \rightarrow \mu \mu e e\right)$. These studies also show a clear displacement of the mass peak due to the energy loss of the electrons. However, the expected separation to the normalization channel $K_{\mathrm{S}}^{0} \rightarrow \pi \pi e e$ with the two pions misidentified as electrons or muons, respectively, combined with the quoted mass resolution, should allow to discriminate between the different channels.

Using the well measured $K_{\mathrm{S}}^{0} \rightarrow \pi \pi e e$ decay as normalization channel, the expected single event sensitivity, assuming no background in the search region, with the $3 \mathrm{fb}^{-1}$ of data accumulated by the LHCb detector is found to be $\sim 10^{-6}\left(\sim 10^{-7}\right)$ for the $K_{\mathrm{S}}^{0} \rightarrow$ eeee $\left(K_{\mathrm{S}}^{0} \rightarrow \mu \mu e e\right)$ channel. Similar studies are being performed with the $K_{\mathrm{s}}^{0} \rightarrow \mu \mu \mu \mu$ channel.

\section{3 $\mathrm{K}^{+}$mass prospects}

Currently experimental results show a disagreement between the two most precise measurements of the $K^{+}$mass [13]. While Denisov et al. measured $m_{K^{ \pm}}=493.696 \pm 0.007 \mathrm{MeV}$ [14], Gall et al. reported $m_{K^{ \pm}}=493.636 \pm 0.011 \mathrm{MeV}$ [15]. LHCb could give a competitive result on this puzzle by means of the $K^{+} \rightarrow \pi^{+} \pi^{-} \pi^{+}$decay.

Due to their large life-time, very few $K^{+}$decay within the first couple of meters of the detector, where their daughters could be reconstructed as long tracks. Thus, the $K^{+}$candidates are reconstructed combining $3 \pi$ from downstream tracks, which have no information from the decay vertex. Random combinations of $3 \pi$ not coming from a decay of a $K^{+}$are easily selected if no requirement on the decay vertex is made. Studies using simulated data at LHCb show a great selection improvement when the tracks are required to be matched to the hits left by the $K^{+}$in the VELO. Figure 4 shows the invariant mass distribution of the $K^{+}$before and after the matching requirement. This technique rejects most of the background with high efficiency on the signal events. More information on this technique can be found in [16]. 



Figure 4: $K^{+}$invariant mass distribution for 2012 data candidates passing hadronic $B$ decay selections. Left: no matching requirement applied. Right: after matching requirement.

A rough estimate of systematic uncertainties shows that the precision on the mass measurement is limited at the moment by statistics. LHC Run 2 may provide enough data to the LHCb detector to allow for a competitive measurement of the $\mathrm{K}^{+}$mass.

\section{$3.4 \Sigma^{+} \rightarrow p \mu^{+} \mu^{-}$prospects}

The HyperCP collaboration at Tevatron reported the observation of three $\Sigma^{+} \rightarrow p \mu^{+} \mu^{-}$events with no background and a branching fraction for this decay of $\left(8.6_{-5.4}^{+6.6} \pm 5.5\right) \times 10^{-8}$ [17]. This result had wide relevance since all the three observed events lie within the expected di-muon mass resolution of $\sim 0.5 \mathrm{MeV} / c^{2}$, possibly pointing towards a $\Sigma^{+} \rightarrow p X^{0}\left(\rightarrow \mu^{+} \mu^{-}\right)$decay. The new intermediate state would have a mass of $214.3 \pm 0.5 \mathrm{MeV} / c^{2}$. The existence of a light neutral particle decaying to a muon pair would have many consequences and several experiments have performed specific tests of this hypothesis [18] [19] [20] [21].

The LHCb collaboration aims for a direct search for the $\Sigma^{+} \rightarrow p \mu^{+} \mu^{-}$decay, taking advantage of the huge amount of $\Sigma^{+}$produced at LHC $p p$ collisions - approximately $40 \%$ of the events contain one. To increase the statistics, downstream tracks could be used in addition to long tracks for this analysis. In the case of downstream tracks, the matching technique presented in section 3.3 would be used to purify the selection. Studies on simulated data show a very good mass resolution for this decay, $\sigma \sim 2 \mathrm{MeV} / c^{2}$. A single event sensitivity of the order of $10^{-8}$ can be reached with the Run 1 dataset $\left(3 \mathrm{fb}^{-1}\right)$.

\section{Summary}

Although the LHCb detector is not designed for strange physics, it has the potential to significantly contribute in the study of this type of decays, taking advantage of the copious production of strange hadrons at the LHC and exploiting the possibility of analyzing data that was triggered as background. A world best upper limit measurement was set for the branching fraction of $K_{\mathrm{S}}^{0} \rightarrow \mu^{+} \mu^{-}$to $9.0 \times 10^{-9}$ at $90 \% \mathrm{CL}$, improving in a factor thirty the previous world best result. After this analysis, strange physics have become a new area of interest for LHCb and several studies are being carried out to asses the sensitivity of some of the most relevant measurements in the 
field. LHCb may provide unique results in the forthcoming years since no other experiment will be looking at $K_{\mathrm{S}}^{0}$ decays in the near future.

\section{References}

[1] S.L. Glashow, J. Iliopoulos and L. Maiani, Weak Interactions with Lepton-Hadron Symmetry, Phys. Rev. D 21285.

[2] J.H. Christenson, J.W. Cronin, V.L. Fitch and R. Turlay, Evidence for the $2 \pi$ Decay of the $K_{2}^{0}$ Meson, Phys. Rev. Lett. 13138.

[3] LHCb collaboration, The LHCb Detector at the LHC, JINST 3 S08005.

[4] LHCb collaboration, Search for the rare decay $K_{\mathrm{S}}^{0} \rightarrow \mu^{+} \mu^{-}$, JHEP 01 (2013) 090.

[5] LHCb collaboration, The LHCb trigger and its performance, JINST 8 P04022.

[6] G. Isidori and R. Unterdorfer, On the short-distance constraints from $K_{\mathrm{L}, \mathrm{S}}^{0} \rightarrow \mu^{+} \mu^{-}$, JHEP 01 (2004) 009.

[7] G. Ecker and A. Pich, The longitudinal muon polarization in $K_{\mathrm{L}}^{0} \rightarrow \mu^{+} \mu^{-}$, Nucl. Phys. B 3661991 189.

[8] S. Gjesdal, G. Presser, P. Steffen, J. Steinberger, F. Vannucci and others, Search for the decay $K_{\mathrm{S}}^{0} \rightarrow 2$ muon, Phys.Lett. B44 (1973) 217-220.

[9] Particle Data Group, Review of Particle Physics, J. Phys. G 37075021.

[10] A.L. Read, Presentation of search results: the CLs technique, J. Phys. G 282693.

[11] NA48 Collaboration, Observation of the rare decay $K_{\mathrm{S}}^{0} \rightarrow \pi^{0} \mu^{+} \mu^{-}$, Phys.Lett. B599 (2004) 197-211.

[12] G. D’Ambrosio, D. Greynat and G. Vulvert, Standard Model and New Physics contributions to $K_{\mathrm{L}}^{0}$ and $K_{\mathrm{S}}^{0}$ into four leptons, Eur.Phys.J. C73 (2013) 2678.

[13] Particle Data Group, Review of Particle Physics, Phys. Rev. D 86010001.

[14] A.S Denisolv et al., New measurements of the mass of the $K^{-}$meson, JETP Lett. 54 (1991) 558-563.

[15] K.P. Gall et al., Precision Measurements of the $K^{?}$ and $\Sigma^{?}$ Masses, Phys. Rev. Lett. 60186.

[16] A. Contu, A method to study long lived charged particles at LHCb, CERN-LHCb-PUB-2014-032.

[17] HyperCP Collaboration, Evidence for the Decay $\Sigma^{+} \rightarrow p \mu^{+} \mu^{-}$, Phys. Rev. Lett. 94021801.

[18] CLEO Collaboration, Search for Very Light CP-Odd Higgs Boson in Radiative Decays of $\Upsilon(1 S)$, Phys. Rev. Lett. 101151802.

[19] KTeV Collaboration, Search for the Rare Decays $K_{\mathrm{L}}^{0} \rightarrow \pi^{0} \pi^{0} \mu^{+} \mu^{-}$and $K_{\mathrm{L}}^{0} \rightarrow \pi^{0} \pi^{0} X^{0} \rightarrow \pi^{0} \pi^{0} \mu^{+} \mu^{-}$, Phys. Rev. Lett. 107201803.

[20] E391a Collaboration, Search for a Light Pseudoscalar Particle in the Decay $K_{\mathrm{L}}^{0} \rightarrow \pi^{0} \pi^{0} X^{0}$, Phys. Rev. Lett. 102051802.

[21] LHCb collaboration, Search for Rare $B_{s}^{0} \rightarrow \mu^{+} \mu^{-} \mu^{+} \mu^{-}$Decays, Phys. Rev. Lett. 110211801. 